\title{
Der grammatische Background der Dramagrammatik
}

Rezension von Hoffmann, Ludger (2013): Deutsche Grammatik. Grundlagen für Lehrerausbildung, Schule, Deutsch als Zweitsprache und Deutsch als Fremdsprache. Berlin: Schmidt.

\section{Maik Walter}

\section{Deutsche Grammatik(en)}

Was haben die folgenden Männer gemeinsam? Jacob Grimm, Wilhelm Wilmanns, Hermann Paul, Friedrich Kaufmann, Wilhelm K. Jude, Johannes Erben, Wolfgang Eichler \& Karl-Dieter Bünting, Gerhard Helbig \& Joachim Buscha, Peter Gallmann \& Horst Sitta, Ulrich Engel, Rudolf Hoberg, und zuletzt Hans Wellmann? Sie alle schrieben eine „Deutsche Grammatik", eine mehr oder weniger umfangreiche Überblicksdarstellung mit identischem Titel, die wohl in der einen oder anderen Form im Buchregal eines jeden Germanisten, Deutschlehrers oder Lehramtsstudenten seinen Platz haben wird.

Nun liegt eine weitere „Deutsche Grammatikmit fast 600 Seiten vor, randvoll mit Beispielen aus der geschriebenen und auch aus der gesprochenen Sprache. Der Untertitel unterscheidet es von seinen Vorgängern und führt gleich vier Adressatenkreise an: Lehrerausbildung, Schule, Deutsch als Zweitsprache und Deutsch als Fremdsprache. LeserInnen von Scenario wären demnach die perfekte Leserschaft. Anders als die gleich lautenden Grammatiken von Helbig/Buscha (2001) oder Engel (1991) ist diese Grammatik primär funktional ausgerichtet, und zwar im Kontext der Sprachvermittlung. Gerade dies sollte die Grammatik zu einem idealen Hilfsmittel für dramagrammatische Projekte (vgl. Even 2001) machen und genau diese Annahme soll im Folgenden geprüft werden.

\section{Funktionale Grammatik}

Aber was heißt eigentlich funktional im Bereich der Grammatik? Geht es nicht immer um das Zusammenspiel von Funktionen und Formen? Beginnen wir deshalb mit dem Funktionalitätsbegriff, einem durchaus schillernden Begriff mit vielen Facetten: Die funktionale Grammatik wird in der Linguistik entweder mit einer kognitiven oder kommunikativen Ausrichtung verbunden (vgl. Welke 
2002, Smirnova \& Mortelmans 2010). Eine grammatische Gesamtdarstellung des Deutschen auf kognitiver Basis existiert bislang nicht, anders sieht es bei den kommunikativ ausgerichteten Grammatiken aus. Für das Deutsche wäre die am Institut für Deutsche Sprache (IDS) in Mannheim (und ebenfalls von Ludger Hoffmann mitherausgegebene) dreibändige Grammatik der deutschen Sprache das derzeitige Standardwerk im Bereich der funktional orientierten Referenzgrammatiken (Zifonun et al. 1997). Jedoch: Wissenschaftliche Grammatiken haben ihre Tücken, denn meist benötigt man ein abgeschlossenes Linguistikstudium, um sie annähernd zu verstehen. Aus der Grammatikbenutzungsforschung weiß man, dass FremdsprachenlehrerInnen nur selten wissenschaftliche Grammatiken zu Rate ziehen (vgl. Walter 2004). Wesentlich seltener, als die Grammatiker es sich gern wünschen. DramapädagogInnen und DeutschlehrerInnen, die bereits einen Blick in die IDS-Grammatik geworfen haben, werden bestätigen, dass sich diese nur bedingt für den Bereich der Fremdsprachvermittlung und insbesondere der Dramagrammatik eignet. Im Alltag greift man als Lehrperson in der Regel zum Lehrwerk und im besten Fall zu bereits für den Unterricht adaptierten Materialien wie den Lernergrammatiken. Ausgesprochen nützlich sind die zum Teil qualitativ hochwertigen frei verfügbaren Ressourcen im Internet wie das ebenfalls am Institut für Deutsche Sprache angesiedelte grammatische Informationssystem grammis (http://hypermedia.ids-mannheim.de), das grammatische Fragen prägnant und verständlich aufbereitet.

Kehren wir zurück zur funktionalen Grammatik. Im Rahmen des kommunikativen Zweigs des Funktionalismus ist in Deutschland die so genannte funktionale Pragmatik ein wichtiger Orientierungspunkt. Darunter versteht man eine linguistische Schule, die auf einem nach dem Bühlerschen Kommunikationsmodell entwickelten gebrauchsorientierten Modell der Sprache basiert und vor allem mit den Linguisten Konrad Ehlich und Jochen Rehbein verbunden ist. Die funktionale Pragmatik mit ihrem Begriffsinventar bildet auch die theoretische Basis der vorliegenden Grammatik. Von der Lektüre profitieren werden deshalb vor allem Lehramtsstudierende, deren linguistische Ausbildung in einem funktionalpragmatischen Hochschulcurriculum verortet ist. Andere Studierende werden viel Zeit investieren müssen, um die Begriffe wie beispielsweise die so genannten „Prozedurenßu verstehen und auf die entsprechende Begrifflichkeit (ob nun der traditionellen Schulgrammatik oder aber moderner Modelle wie die des generativen Frameworks) zu transferieren. SprachlehrerInnen werden wohl kaum die Zeit aufwenden, sich in das Modell einzuarbeiten, um gezielt Antworten auf Fragen zu finden. Mit bereits etablierten Angeboten wie dem oben bereits genannten grammatischen Informationssystem grammis wird man hier besser bedient.

\section{Die Hoffmannsche Grammatik im Überblick}

Die „Deutsche Grammatik"besteht aus 8 Kapiteln und einem Serviceteil. Die Länge der einzelnen Kapitel schwankt zwischen 11 und 152 Seiten. Zum 
Anfang der 8 Kapitel gibt es jeweils einen „didaktischen Kommentar", der den Inhalt des Kapitels in wenigen Worten zusammenfasst, sowie weiterführende Literaturhinweise. In 6 Kapiteln werden darüberhinaus zu Beginn die zentralen „Kategorienünd in drei Kapiteln die „Prozedurenängeführt. Für das Kapitel F „Gedanken verknüpfen und erweiternßind dies „Konnektivpartikel und Konjunktorßowie die „operative und symbolische Prozedur"(S. 419). In den entsprechenden Unterkapiteln werden ebenfalls zum Teil „didaktische Kommentare", „Prozedurenünd „Kategorienängeführt und etwas verwirrend auch die „Wortarten", die in anderen Fällen als „Kategorieërfasst wurden. Aufgaben und Projektideen für die Schule runden die Kapitel ab.

Im ersten Kapitel wird die begriffliche Basis, und das meint im Wesentlichen das Modell der funktionalen Pragmatik, auch mit den entsprechenden Literaturhinweisen vorgestellt. Wichtig ist hier vor allem der Begriff der sprachlichen Prozedur, der kleinsten funktionalen Einheit. Das zweite Kapitel thematisiert die Zuordnung von Formen und Funktionen, bevor dann in den folgenden acht Kapiteln der Kernbereich der deutschen Grammatik dargestellt wird. Der Formulierung von Redegegenständen (C) und der Gedanken (D), folgen deren Ausbau (E) und deren Verknüpfung und Erweiterung (F). In den letzten beiden Kapiteln wird die Abfolge und Kommunikative Gewichtung (G) sowie die Zweckbereiche des Handelns und Äußerungsmodi (H) dargestellt. Ein ServiceKapitel, in dem neben sprachlichen Testverfahren unter anderem auch ein Portrait des für die Kontrastierung an einigen Stellen herangezogenen Türkisch enthalten ist, schließt das Buch ab. Für den Fremdsprachenkontext ist der Bezug $\mathrm{zu}$ anderen Sprachen durchaus mit Gewinn zu lesen. Beispielsweise wenn die Raumrelationen im Türkischen und mögliche Konsequenzen für Lerner des Deutschen mit der L1 Türkisch vorgeführt werden (S. 381-384). In vielen Fällen kann der kontrastive Zugang als Anregung dienen, eigene Recherchen mit der entsprechenden L1 durchzuführen. Für sprachlich heterogene Lernergruppen hingegen wäre darüber nachzudenken, ob ein stärker typologischer Zuschnitt nicht angemessener wäre.

Im Kapitel zur „Formulierung der Gedanken"(S. 227-324) geht es um den Dreh- und Angelpunkt einer jeden Grammatik, um die Behandlung der Verben und der entsprechenden Mitspieler. Nachdem der Formenreichtum des Verbalparadigmas dargestellt wurde, greift Ludger Hoffmann auf die Theatermetapher der Szene zurück (S. 309), die vor allem durch die Valenztheorie populär wurde und die Klaus Welke sehr treffend auch als eine der beiden Gründungsmetaphern der Valenztheorie bezeichnet (vgl. Welke 2011: 44). Der Aufbau des Satzes wurde hierbei als eine Szene im Theater betrachtet, bei der es Handelnde (Aktanten) und Requisiten (Circumstanten) gibt, die gemeinsam das Geschehen strukturieren. Stark vereinfacht ausgedrückt, eröffnet das Verb den Rahmen, in dem Subjekt und Objekte, umgeben von den Adverbialbestimmungen, auftreten. Linguisten, Sprachlehrer und DaF-Studierende wissen um die Schwierigkeiten einer geglückten Aufführung eines Satzes, zumal wenn sie auch noch zusätzlich in einer fremden Sprache vollzogen wird. Solche Metaphern könnten auch für 
eine Umsetzung in der Dramagrammatik ein gute Inspiration sein.

Wichtiger als die Metaphorik ist für den Dramagrammatiker jedoch die Aufbereitung der Hintergrundinformationen, die für eine entsprechende dramagrammatische Einheit benötigt wird. Von weitaus größerem Interesse dürften Fragen nach dem sprachlichen Inventar zum Ausdruck bestimmter Funktionen und deren Systematik sein wie beispielsweise nach dem Ausdruck der Ungewissheit im Deutschen. Oder aber nach Gebrauchsdifferenzen zwischen sprachlichen Mitteln wie etwa die Unterschiede zwischen Konjunktiv I und II und nach dem Zusammenhang mit den interagierenden, bzw. konkurrierenden Modalausdrücken wie vermutlich oder möglicherweise. Während man beim Konjunktiv in der Grammatik schnell ans Ziel gelangt und sich dann durch die verschiedenen Belege arbeiten kann, ist dies bei den Modalwörtern (vermutlich, möglicherweise,...) kein leichtes Unterfangen: Weder im Wortregister noch im Inhaltsverzeichnis sind sie zu finden. Behandelt werden sie im Abschnitt „Modalisieren", in dem sie als Modalpartikeln auftauchen (S. 400). Diese Kategorisierung ist linguistisch nicht unumstritten, soll aber hier nicht weiter verfolgt werden. Ist man beim gesuchten Ausdruck angelangt, findet man reichhaltiges Material.

Die Grammatik kann dennoch als Nachschlagewerk für dramagrammatisches Arbeiten nicht empfohlen werden, vielmehr ist sie eine Lesegrammatik, die sehr viel Zeit erfordert. Begibt man sich mit Ludger Hoffmann, dem passionierten Grammatiker, auf diese Reise, wird man staunen über mögliche Finessen in der Sprache und kann sich von seinen Fundstücken und Beschreibungen inspirieren lassen. Nicht nur die typischen Vertreter, sondern auch die Randbereiche der Sprache werden hier diskutiert und an vielen Stellen mit einer Analyse von Lernertexten versehen. Diesen Zusammenstellungen und Analysen zu folgen, macht Spaß und einige Entdeckungen warten hier auf die LeserInnen.

\section{Eine „Deutsche Grammatikfür die Dramagrammatik?}

Ist diese Grammatik nun für die Dramagrammatik geeignet? Wer gezielt Antworten auf grammatischeFragen sucht, wird mit diesem Buch nicht zufrieden sein. Für Fragen zum formalen Inventar und der Systematik sprachlicher Strukturen wird man weiterhin aufdie mitVollständigkeitsanspruch angetretene Grammatik von Helbig/Buscha angewiesen sein. Die Warnung des Verfassers im Vorwort „Die Grammatik bedarf zusammenhängender Lektüre, damit ein Bild des Deutschen entstehen kann"(S. 5) sollte durchaus ernst genommen werden. Wer jedoch ein breites, funktional pragmatisch gezeichnetes Panorama der deutschen Sprache vorgeführt bekommen will, wird derzeit kein besseres Buch zum Deutschen finden.

\section{Bibliografie:}

Engel, Ulrich (1991): Deutsche Grammatik. 2. Aufl. Heidelberg: Groos 
Even, Susanne (2003): Drama Grammatik. Dramapädagogische Ansätze für den Grammatikunterricht Deutsch als Fremdsprache. München: iucidium

Helbig, Gerhard; Buscha, Joachim (2001): Deutsche Grammatik. Ein Handbuch für den Ausländerunterricht. Berlin, München, Wien, Zürich, New York: Langenscheidt

Smirnova, Elena; Mortelmans, Tanja (2010): Funktionale Grammatik. Konzepte und Theorien. Berlin; New York: de Gruyter (De Gruyter Studium)

Welke, Klaus (2002): Deutsche Syntax funktional. Perspektiviertheit syntaktischer Strukturen. Tübingen: Stauffenburg Verlag (Stauffenburg Linguistik, 22)

Welke, Klaus (2011): Valenzgrammatik des Deutschen. Eine Einführung. Berlin; New York: de Gruyter (De Gruyter Studium)

Walter, Maik (2004): Grammatisches Hintergrundwissen für DaF. Oder warum Philosophen nicht die besseren Mediziner sind. In: Lüger, Heinz-Helmut; Rothenhäusler, Rainer (Hg.): Linguistik für die Fremdsprache Deutsch. Landau: Verlag Empirische Pädagogik (Beiträge zur Fremdsprachenvermittlung Sonderheft 7), 53-84

Zifonun Gisela; Hoffman, Ludger; Strecker, Bruno (Hg.) (1997): Grammatik der deutschen Sprache. 3 Bände. Berlin; New York: Walter de Gruyter (Schriften des Instituts für deutsche Sprache 7) 